\title{
Seed priming mitigates the effects of saline-alkali stress in soybean seedlings
}

Ling Yan Dai ${ }^{1}$, Hong De Zhu ${ }^{2}$, Kui De Yin ${ }^{1}$, Ji Dao Du ${ }^{2}$ Yu Xian Zhang ${ }^{2 *}$

\section{ABSTRACT}

Soda saline-alkali soil has double adverse effects on growth, morphogenesis and yield of the crops by creating a salinity stress and a high $\mathrm{pH}$ value in the rhizosphere surroundings of the plant. Seed priming can be an effective approach to enhance stress adaptation in seedlings growing in salinity stressed conditions. The present study examined the role of hydropriming and comprehensive seed priming with $\mathrm{ZnSO}_{4}, \mathrm{CaCl}_{2}$, betaine hydrochloride and $\mathrm{GA}_{3}$ to enhance the soda saline-alkali tolerance in soybeans (Glycine max [L.] Merr.) seedlings. An unprimed control treatment was also maintained for comparison. The results show that two seed priming treatments can effectively mitigate the negative effects of soda salinealkali stress. Nevertheless, compared with hydropriming, soybean seedlings from the comprehensive seed priming treatment had better growth performance through accumulation of soluble sugar and free proline contents, enhancing the activities of superoxide dismutase (SOD) and catalase (CAT), decreasing the malondialdehyde (MDA) content formed from lipid peroxidation, increasing the photosynthetic pigment contents, maintaining a better membrane integrity of the chloroplasts and mitochondria, and generating more starch grains in the chloroplasts of spongy mesophyll cells under soda saline-alkali stress. In conclusion, our results suggest that soybean seedlings from comprehensive seed priming exhibited a higher tolerance to soda saline-alkali stress than the unprimed control treatment.

Key words: Comprehensive seed priming, Glycine max, hydropriming, physiological property, tolerance.

${ }^{1}$ Heilongjiang Bayi Agricultural University, College of Life Science and Technology, Xinyang Road 2\#, 163319, Heilongjiang Province, China. ${ }^{2}$ Heilongjiang Bayi Agricultural University, College of Agronomy, Xinyang Road 2\#, 163319 Daqing, Heilongjiang Province, China. *Corresponding author (zyx_lxy@126.com).

Received: 16 November 2016.

Accepted: 26 March 2017

doi:10.4067/S0718-58392017000200118

\section{INTRODUCTION}

Globally, soil salinization is one of the most important ecological issues of dryland agriculture and has become a major hindrance for the (high) yield of crops. Moreover, salinization is spreading in irrigated lands because of inappropriate management of irrigation and farming (Abraha and Yohannes, 2013). Among them, inland soda saline-alkali soil (with $\mathrm{Na}_{2} \mathrm{CO}_{3}$ and $\mathrm{NaHCO}_{3}$ as the main component of salinity) has a high $\mathrm{pH}$ value except for excessive $\mathrm{Na}^{+}$ions, which has more inapposite physical and chemical properties and a more serious harm to crops. Soda saline-alkali soil has double the adverse effects on the seedling growth of crops by creating salinity stress and high $\mathrm{pH}$ value in the rhizosphere surroundings of the plant (Dai et al., 2012). Soil salinity may affect plant growth not only by creating a lower osmotic potential external to the plant and preventing water uptake but also through the toxic effects of $\mathrm{Na}^{+}$and $\mathrm{Cl}^{-}$ions on the plant. A high $\mathrm{pH}$ environment can cause $\mathrm{Ca}, \mathrm{Mn}, \mathrm{P}, \mathrm{Fe}$ and other elements to be consolidated by soil particles and lead to plant difficulty absorb them, it can also destroy various acids of the crop root environment, and directly corrode roots of the young plant. In summary, salinity causes a series of biochemical, physiological and metabolic changes in plants (Paparella et al., 2015; Ibrahim, 2016), which may result in oxidative stress and affect plant metabolism, performance and yield (Shafi et al., 2009). Therefore, increasing the crop resistance to all types of biotic/abiotic stresses is the key to enhancing seed quality and achieving rapid and uniform seedling emergence.

Recently, many strategies have been being applied to induce abiotic stress tolerance in crops based on genetic control and physiological approaches. Among the various strategies, seed priming, which is easy, low-cost and low risk, is an effective and practical technique to enhance rapid and uniform emergence, high seedling vigor, and better yields in many field crops, especially under an adverse environment (Ashraf and Foolad, 2005; Paparella et al., 2015). Seed priming is a controlled hydration process that is followed by redrying and triggers many of the physiological processes associated with the early phase of germination and prepares the seed for radicle protrusion (Paparella et al., 2015). Moreover, it can decrease the physical resistance of the endosperm during imbibition and repair membranes but also lead to the development of immature embryos and leach emergence inhibitors (Ibrahim, 2016). Generally, various seed priming techniques, which include hydropriming, osmopriming, chemical priming, nutrient priming, hormonal-priming, and 
redox priming, are used to induce pre-germination changes (Paparella et al., 2015; Hussain et al., 2016). Currently, some studies have proven that seed priming is a useful physiological approach for making glycophyte species adapt to saline conditions such as hot peppers (Khan et al., 2009), maize (Abraha and Yohannes, 2013), tomatoes (Pradhan et al., 2014) and soybeans (Miladinov et al., 2015).

For many crops, two stages, seed germination and seedling growth, are critical for establishment and the most sensitive to abiotic stress (Patade et al., 2011). Presently, considerable research has focused on effects of seed priming under different salinity conditions (Hussain et al., 2016). However, little is known regarding the effect on the plant used by seed priming under soda saline-alkali stress. Therefore, in the present study, we examined hydropriming and multiple chemical priming against soda saline-alkali stress in soybean seedlings. The effective reagents were chosen and their concentrations and priming methods were pre-optimized in various preliminary studies based on soybean germination and seedling growth performance (Dai et al., 2015). Additionally, the physiological and biochemical changes were unraveled in soybean seedlings under the influence of seed priming and soda saline-alkali stress to obtain a better understanding of priming-induced mechanisms.

\section{MATERIALS AND METHODS}

\section{Plant material}

Soybean (Glycine max [L.] Merr.) cv. Nematode resistance No. 10 was used in the present experiment. The 'Nematode resistance No. 10' is widely cultivated by soybean farmers in soda saline-alkali land of Heilongjiang Province in China. The initial germination and moisture content of seeds was $>95 \%$ and $8 \%$ (on the basis of dry weight), respectively.

\section{Priming treatment, stress treatment and growth}

The seed-priming treatments were hydropriming (HP; distilled water) and comprehensive seed priming (CP; $0.025 \% \mathrm{ZnSO}_{4} \cdot 7 \mathrm{H}_{2} \mathrm{O}, 52.5 \mathrm{mM} \mathrm{L} \mathrm{CaCl}_{2} \cdot 2 \mathrm{H}_{2} \mathrm{O}, 0.5 \mathrm{~g} \mathrm{~L}^{-1}$ betaine hydrochloride and $30 \mathrm{mg} \mathrm{L}^{-1} \mathrm{GA}_{3}$ ). An unprimed control (UP), namely, dry seeds were maintained for a comparison. The seeds were primed in the distilled water and comprehensive solution, respectively, in the dark at $25^{\circ} \mathrm{C}$ for $2 \mathrm{~h}$. The ratio of seed volume to solution volume (v/v) was approximately 4:5. Thereafter, the seed was washed with distilled water 3 times and then surface-dried using blotting papers. Finally, the treated seed was dried back to its original moisture content at room temperature (approximately $25^{\circ} \mathrm{C}$ and $45 \% \mathrm{RH}$ ).

Twenty seeds were planted in sand-filled plastic pots $(20.5 \times 22.0 \mathrm{~cm})$, which were pre-irrigated with Hoagland nutrient solution (HNS) and re-irrigated every $2 \mathrm{~d}$. After germinating, all of the seedlings (UP, HP and CP) were treated with a soda saline-alkali solution. The stress solution was made of $\mathrm{NaHCO}_{3}$ and $\mathrm{Na}_{2} \mathrm{CO}_{3}$ (in a molar ratio of $5: 1$ ), which is a $10 \mathrm{mM} \mathrm{L}^{-1}$ final concentration, $\mathrm{pH} 9.07$, and $1.02 \%$ salinity and were prepared in HNS. The soda saline-alkali stress lasted for $14 \mathrm{~d}$. For each treatment, five pots containing seedlings under a similar growth situation were randomly selected, with each pot representing a single replicate.

\section{Plant growth parameters determination}

After exposure to soda saline-alkali stress treatment for 14 $\mathrm{d}$, seedlings were randomly sampled from each treatment. The shoots and roots were separated. The shoot height, root length, shoot weight and root weight of five plants from each treatment were determined. A total of five roots were selected from each treatment using the triphenyltetrazolium chloride (TTC) method for determining root vigor (Zheng et al., 2016). Root vigor was determined as reduced TTC per root fresh weiht per hour ( $\mathrm{Mg}_{\mathrm{TTC}} \mathrm{g}^{-1} \mathrm{FW} \mathrm{h}^{-1}$ (Guo et al., 2015).

Root vigor $=$ Reduced TTC $(\mu \mathrm{g}) /$ Root weight $(g) \times$ Time $(h)$

\section{Physiological parameters of leaves}

Soluble proteins were extracted from young leaves and the content was determined according to the method of Bradford (1976). The amount of protein was conducted with reference to a standard curve obtained from bovine serum albumin. The soluble protein contents were expressed as $\mathrm{mg} \mathrm{g}^{-1} \mathrm{FW}$ leaves.

Proline was determined based on the method described by Hussain et al. (2016). The proline content in leaves was estimated by referring to a standard curve of L-proline. The free proline content was expressed as $\mu \mathrm{g} \mathrm{g}^{-1} \mathrm{FW}$ leaves.

The soluble sugars were measured following Nazarli and Faraji (2011). The content of soluble sugar was determined using a glucose standard and expressed as $\mathrm{mg} \mathrm{g}^{-1} \mathrm{FW}$ leaves.

The pigments of the leaves, including chlorophyll $\mathrm{a}$, chlorophyll b and carotenoids, were extracted with $80 \%$ acetone and quantified following the method of Lichtenthaler (1987). The pigment content was expressed as $\mathrm{mg} \mathrm{g}^{-1} \mathrm{FW}$ leaves.

\section{Antioxidant enzyme activities and lipid peroxidation}

The activities of superoxide dismutase (SOD) and catalase (CAT) were determined according to the methods provided in Hussain et al. (2016). A SOD and CAT detection kits were purchased from Nanjing Jiancheng Bioengineering Institute (Nanjing, Jiangsu, China). The superoxide anion radicals are produced from xanthine oxidized by the xanthine oxidase. One unit of SOD activity was defined as the amount of extract that provides $50 \%$ inhibition in a reduction of xanthine when monitored at $550 \mathrm{~nm}$ (Infinite M200, Tecan Group, Männedorf, Switzerland) and was expressed as $\mathrm{U} \mathrm{g}^{-1} \mathrm{FW}$. One unit of CAT activity was defined as the amount of enzyme that 
will decompose $1 \mu$ mol $\mathrm{H}_{2} \mathrm{O}_{2}$ at $405 \mathrm{~nm}$ (Infinite $\mathrm{M} 200$, Tecan) per second and was expressed as $\mathrm{U} \mathrm{g}^{-1} \mathrm{FW}$.

The peroxidase (POD) activity was determined by the method of guaiacol oxidation following the method of Zheng et al. (2015). Readings at $470 \mathrm{~nm}$ (Infinite M200) were recorded within 3 min after the start of the reaction at 1 min intervals. One unit of POD activity was defined as an absorbance change of 0.01 units per min and was expressed as $\mathrm{Ug}^{-1} \mathrm{FW}$.

The lipid peroxidation in soybean seedling leaves was determined as malondialdehyde (MDA) content using the thiobarbituric acid method.

\section{Transmission electron microscopy}

The same leaves were used to conduct an ultrastructural analysis according to the methods provided in Guo et al. (2008). The median region of the fully expanded leaves of each treatment, which was away from the midrib, was collected (approximately $4 \mathrm{~mm}^{2}$ ). After fixation in glutaraldehyde, the samples were post-fixed in osmium tetroxide, dehydrated in a series of acetone and $100 \%$ alcohol (two times; 60 min each), and embedded in Epon 812 resin. The samples were then cut into thin sections with a UC7 Leica ultramicrotome (Germany), stained on copper grids with lead citrate and uranyl acetate, and observed in a transmission electron microscope (H-7650, Hitachi, Tokyo, Japan) operating at $75 \mathrm{kV}$.

\section{Statistical analysis}

The data were statistically analyzed with ANOVA using the SPSS software program Version 13.0 (SPSS, Chicago, Illinois, USA). The statistical significance was assessed using Duncan's multiple range test at $P<0.05$. The significance analyses were conducted to compare the effects of the different treatments (UP, HP and CP) on each parameter.

\section{RESULTS}

\section{Plant growth parameters}

The data regarding plant growth of unprimed (UP), hydroprimed (HP) and comprehensive seed primed (CP) seeds of soybeans under soda saline-alkali stress are shown in Table 1. Compared with HP and CP, shoot height, root length, shoot weight, root weight and root vigor of soybean seedlings from UP seeds were all decreased, respectively. Shoot height, root length and weight, and root vigor of soybean seedlings from CP seeds were significantly higher than those from UP seeds, except for the shoot weight. Although shoot height and weight, root length, and root vigor of soybean seedling from HP seeds increased, they did not increase significantly compared with UP. All of the growth parameters of seedlings from HP seeds were lower than $\mathrm{CP}$ but were remarkably decreased for shoot height and root vigor.

\section{Soluble protein, soluble sugar and proline}

The soluble proteins of soybean seedlings were significantly similar among UP, HP and CP seeds under soda salinealkali stress (Table 2). For soluble sugar, soybean seedlings from primed seeds (HP and CP) were remarkably higher than UP. Compared with UP, accumulation of free proline in the seedlings from the primed seeds increased prominently. Moreover, the proline content in the CP seedlings was significantly higher than in HP seedlings.

\section{Antioxidant system components and MDA}

The SOD, POD, and CAT activities and MDA content of soybean seedlings under soda saline-alkali stress are presented in Table 3. The POD activities of seedlings had no significant difference among UP, HP and CP. SOD and CAT activities of seedlings from CP seeds were remarkably increased compared to HP and UP. All of the seedlings from the primed seeds exhibited a lower accumulation of MDA than UP. In all of the treatments, the seedlings from CP had the lowest MDA content and amounted to $55.96 \%$ of the MDA accumulation in UP.

\section{Photosynthetic pigments}

The leaf concentrations of chlorophyll a, chlorophyll b, carotenoids and total pigment in soybean seedlings from HP and CP under soda saline-alkali stress were significantly increased compared to UP (Figure 1). Moreover, several types of pigments of seedlings in HP were prominently

Table 2. Effect of unpriming control (UP), hydro-priming (HP) and comprehensive seed priming (CP) on soluble protein, soluble sugar and proline of soybean seedling leaves under soda saline-alkali stress.

\begin{tabular}{llcc}
\hline Treatments & \multicolumn{1}{c}{ Soluble protein } & Soluble sugar & \multicolumn{1}{c}{ Proline } \\
\cline { 2 - 3 } & \multicolumn{2}{c}{$\mathrm{mg} \mathrm{g}^{-1} \mathrm{FW}$} & $\mu \mathrm{g} \mathrm{g}^{-1} \mathrm{FW}$ \\
$\mathrm{UP}$ & $18.08 \pm 0.03 \mathrm{a}$ & $0.51 \pm 0.01 \mathrm{~b}$ & $322.91 \pm 8.35 \mathrm{c}$ \\
$\mathrm{HP}$ & $17.98 \pm 0.18 \mathrm{a}$ & $0.61 \pm 0.01 \mathrm{a}$ & $454.41 \pm 8.79 \mathrm{~b}$ \\
$\mathrm{CP}$ & $18.28 \pm 0.44 \mathrm{a}$ & $0.63 \pm 0.02 \mathrm{a}$ & $529.27 \pm 16.43 \mathrm{a}$ \\
\hline
\end{tabular}

Mean $\pm(\mathrm{SD})$ values followed by the same letters within each column are nonsignificantly different at 0.05 (ANOVA and Duncan's multiple range test) $\mathrm{n}=3$

Table 1. Effect of unpriming control (UP), hydro-priming (HP) and comprehensive seed priming (CP) on shoot height, root length, shoot weight, root weight and root vigor of soybean seedlings under soda saline-alkali stress.

\begin{tabular}{|c|c|c|c|c|c|}
\hline Treatments & Shoot height & Root length & Shoot weight & Root weight & Root vigor \\
\hline & 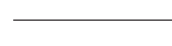 & 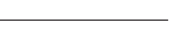 & 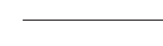 & - & $\mu \mathrm{g} \mathrm{g}^{-1} \mathrm{FW} \mathrm{h}^{-1}$ \\
\hline UP & $10.40 \pm 0.58 b$ & $9.10 \pm 0.31 b$ & $1.29 \pm 0.20 a$ & $0.38 \pm 0.07 b$ & $22.28 \pm 1.49 b$ \\
\hline $\mathrm{HP}$ & $11.05 \pm 1.33 b$ & $9.66 \pm 0.95 \mathrm{ab}$ & $1.36 \pm 0.16 a$ & $0.50 \pm 0.07 a$ & $24.06 \pm 0.49 b$ \\
\hline $\mathrm{CP}$ & $12.30 \pm 1.05 \mathrm{a}$ & $10.16 \pm 0.61 \mathrm{a}$ & $1.48 \pm 0.36 \mathrm{a}$ & $0.55 \pm 0.11 \mathrm{a}$ & $45.32 \pm 1.03 \mathrm{a}$ \\
\hline
\end{tabular}

Mean $\pm(\mathrm{SD})$ values followed by the same letters within each column are nonsignificantly different at 0.05 (ANOVA and Duncan's multiple range test) $\mathrm{n}=5$ 
Table 3. Effect of unpriming control (UP), hydro-priming (HP) and comprehensive seed priming $(\mathrm{CP})$ on superoxide dismutase (SOD), peroxidase (POD), catalase (CAT) and malondialdehyde (MDA) of soybean seedling leaves under soda saline-alkali stress.

\begin{tabular}{lcccc}
\hline Treatments & \multicolumn{1}{c}{ SOD } & POD & CAT & MDA \\
\cline { 2 - 4 } & & $\mathrm{U} \mathrm{g}^{-1} \mathrm{FW}$ & $\mathrm{nmol} \mathrm{g}{ }^{-1} \mathrm{FW}$ \\
$\mathrm{UP}$ & $393.22 \pm 41.52 \mathrm{~b}$ & $1439.47 \pm 80.43 \mathrm{a}$ & $43.53 \pm 10.59 \mathrm{c}$ & $89.62 \pm 2.65 \mathrm{a}$ \\
$\mathrm{HP}$ & $424.26 \pm 47.65 \mathrm{~b}$ & $1406.27 \pm 96.51 \mathrm{a}$ & $70.88 \pm 14.71 \mathrm{~b}$ & $69.77 \pm 4.62 \mathrm{~b}$ \\
$\mathrm{CP}$ & $526.09 \pm 51.16 \mathrm{a}$ & $1554.32 \pm 26.66 \mathrm{a}$ & $134.41 \pm 6.47 \mathrm{a}$ & $50.15 \pm 4.55 \mathrm{c}$
\end{tabular}

Mean $\pm(\mathrm{SD})$ values followed by the same letters within each column are nonsignificantly different at 0.05 (ANOVA and Duncan's multiple range test) $\mathrm{n}=3$.

lower compared to those in $\mathrm{CP}$. The leaf concentrations of chlorophyll a, chlorophyll b, carotenoids and total pigment were higher in soybean seedlings from CP by $44.4 \%$, $47.3 \%, 51.4 \%$, and $46.8 \%$, respectively, compared to those from UP.

\section{Mesophyll cell ultrastructure}

To study the damage of chloroplasts and other organelles of mesophyll cells in soybean seedlings caused by soda saline-alkali stress, the ultrastructure of three treatments was investigated with a transmission electron microscope (Figure 2). The chloroplasts were similar in UP, HP and $\mathrm{CP}$ and some exhibited ellipsoidal shapes, whereas some showed subrotund shapes. The chloroplasts appeared to be swollen with little granal stacking and few intergranal lamellae. Some chloroplast granal stacks were distorted, indistinct or separated from intergranal lamellae. All of the starch grains laid in a similar orientation to the chloroplast while kept away from the cell wall. From UP and HP treatments, the plasma membrane of spongy mesophyll cells was withdrawn from the cell wall, the chloroplasts appeared to be partially disintegrated, and several plastoglobuli were presented in the chloroplasts and some appeared to release their contents into the cytoplasm (Figures 2A and 2C). Compared with HP, the chloroplast was more dilated and disintegration of the chloroplast membrane was more serious in UP (Figures 2B and 2D). The mitochondria appeared distended with a degenerated envelope, and some dispersed cristae were exhibited in the mitochondrial matrix in HP (Figure 2D). Although quantitative measurements were not conducted, it was obvious that spongy mesophyll cells from CP possessed more starch grains (Figure 2E) and fewer plastoglobuli (Figure 2F) than those from UP (Figures $2 \mathrm{~A}$ and $2 \mathrm{~B}$ ) and $\mathrm{HP}$ (Figures $2 \mathrm{C}$ and 2D).

\section{DISCUSSION}

Seed germination and seedling establishment were inhibited or delayed by salt and osmotic stresses. For plants, the soda saline-alkali stress can cause salt, osmotic and high $\mathrm{pH}$ value stresses. To relieve damage from soybeans grown in saline and alkaline land, we adapted a seed-priming technology to improve soybean seedling resistance. The optimal concentration of calcium chloride, zinc sulfate, betaine hydrochloride, and gibberellic acid $\left(\mathrm{GA}_{3}\right)$ were mixed in our priming agent, which was integrated and included inorganic salt, organics and hormones (Dai et al., 2015). Misra et al. (2001) reported that in mung bean and Brassica seedlings, the maximum Chla fluorescence level $\left(\mathrm{F}_{\mathrm{M}}\right)$, quantum yield of the primary photochemistry of PSII $\left(\phi_{\mathrm{Po}}\right)$, quantum yield of electron transport beyond $\mathrm{QA}^{-}$decreased significantly with $\mathrm{NaCl}$ treatments after 3 $\mathrm{d}$ of treatment. But, Addition of $\mathrm{CaCl}_{2}$ along with $\mathrm{NaCl}$ could completely ameliorate the inhibitory effect of $\mathrm{NaCl}$. Additionally, the proline content of priming pepper (Capsicum annuum L.) seeds with $\mathrm{CaCl}_{2}$ caused a marked increase under salt stress (Aloui et al., 2014). The crops planted in the soda saline-alkali soil can reduce their yields. However, in the $\mathrm{Zn}$ deficient soil of Pakistan, wheat and chickpea seeds primed with $\mathrm{ZnSO}_{4}$ can increase grain yield associated with increases in total biomass (Harris et al., 2008). Glycine betaine is an important organic osmolyte accumulated in many of plants to respond to abiotic stress. Priming pepper (C. апnиит) seed with glycine betaine revealed a significant decrease in MDA content and enhancement in proline content and SOD enzyme activity under salinity stress conditions (Korkmaz and Şirikçi,

Figure 1. Effect of unpriming control (UP), hydro-priming (HP) and comprehensive seed priming (CP) on chlorophyll a, chlorophyll b, carotenoids and total pigment content of soybean seedlings under soda saline-alkali stress.

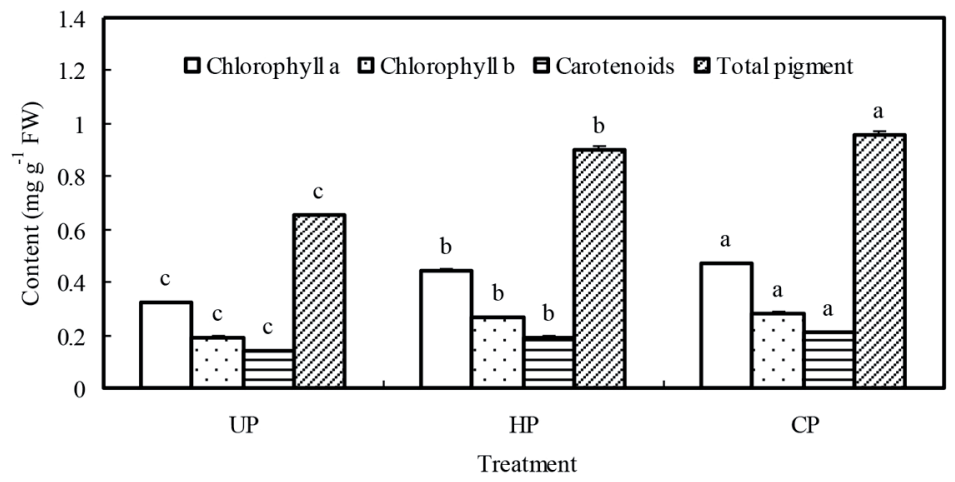

Note: The total pigment content is Chla $+\mathrm{Chlb}+$ carotenoids. Means indicated by different letters are significantly different at the $\mathrm{p}<0.05$ level as determined using Duncan's multiple range test. 
Figure 2. Effect of unpriming control (UP), hydro-priming (HP) and comprehensive seed priming (CP) on mesophyll cell ultrastructure of soybean seedlings under soda saline-alkali stress.
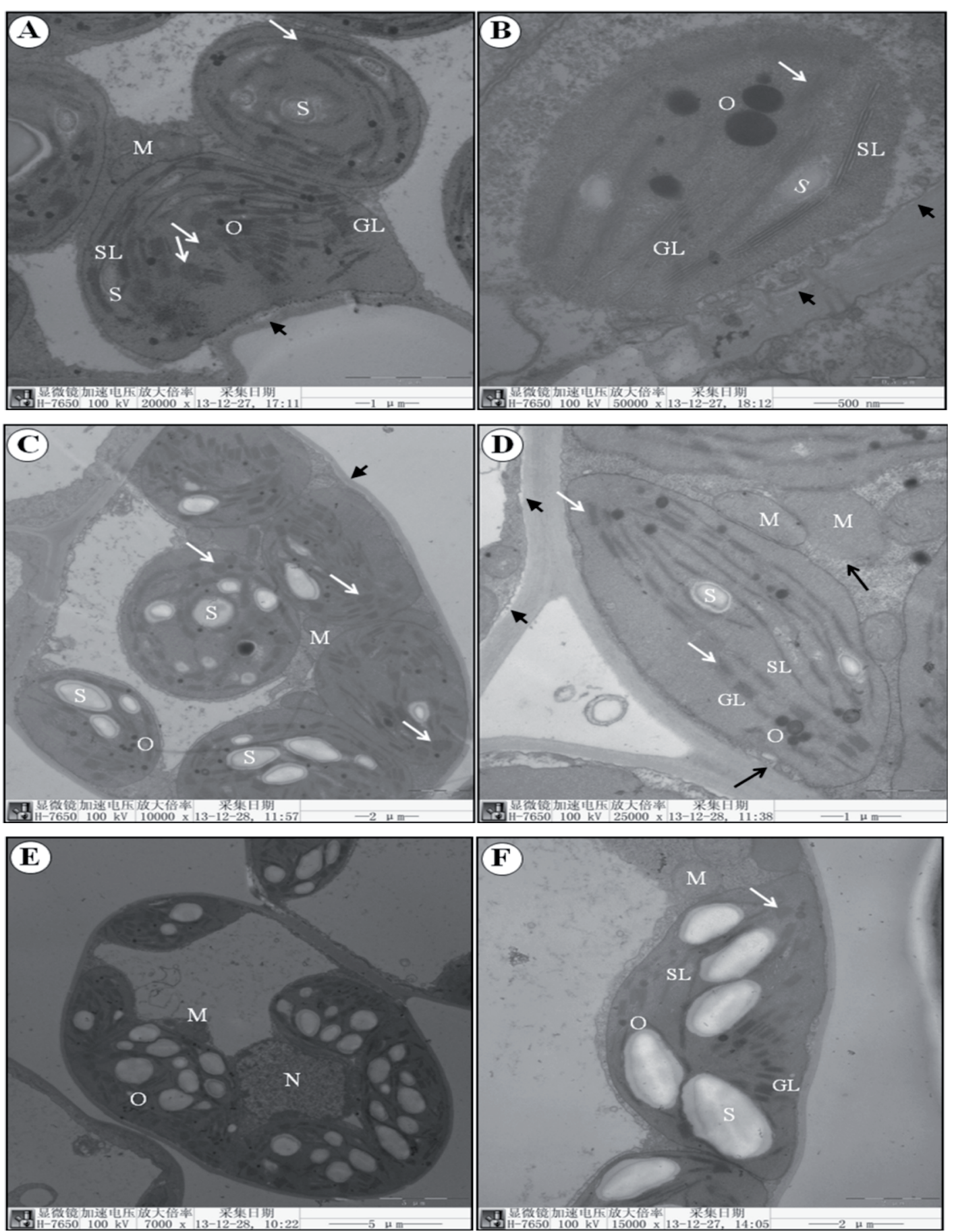

S: Starch grains; O: osmiophilic droplet; SL: stroma lamella; GL: grana lamella; M: mitochondria; N: nucleus. Transmission electron microscopy (TEM) of spongy mesophyll cells of leaves from UP (A, B); from HP (C, D); and from $\mathrm{CP}$ (E, F). Note that plasma membrane of mesophyll cells was withdrawn from the cell wall (short black arrows). Note that the membrane was disintegrated from chloroplast and mitochondrion (black arrows). Note that the granal stacks and thylakoids were abnormal or indistinct (white arrows).

2011). Gibberellins can inhibit abscisic acid (ABA) activity by activating the ABA-catabolizing enzymes and decreasing ABA accumulation during germination and then enhance seed germination (Miransari and Smith, 2014). GA 3 priming can improve seed germination and increase salt tolerance of alfalfa through enhancing the activities of antioxidant enzymes and reducing membrane damage as estimated using a reduction of electrolyte leakage and MDA level in salt stressed seedlings (Younesi and Moradi, 2015).

The results indicate that plant seedling growth, which is adversely influenced by salinity stress, is due to a reduction in germination and inhibition of seedling establishment 
(Kaya et al., 2006). The radicle and stem length of pepper were reduced by increased salinity levels, and plants derived from primed seeds developed a longer radicle and stem for all salinity levels and for all cultivars (Aloui et al., 2014). These results are in accordance with Liu et al. (1996), who reported that osmotic priming can improve radical and plumule length in primed tomato seeds. In this study, similar results were concluded, that is, shoot height, root length, shoot weight, root weight and root vigor of soybean seedlings from primed seeds were higher than those from unprimed seeds under soda saline-alkali stress (Table 1). Moreover, compared with HP, the soybean seedlings from $\mathrm{CP}$ seeds expressed better growing status. The soybean seedlings seeds primed with $\mathrm{ZnSO}_{4}, \mathrm{CaCl}_{2}$, betaine hydrochloride and $\mathrm{GA}_{3}$ might more preferably absorb water and nutrients attributed to stronger roots surrounded by soda saline-alkali conditions.

Under soda saline-alkali stress, it is very difficult for plants to absorb water from their surroundings as a result of the lower water potential. Plants accumulate inorganic or synthesis of organic solutes, such as free proline and soluble sugar, as osmotic adjustments in response to the decreased external water potential and to resist salinity-induced osmotic stress (Zhu et al., 2011).

Our results showed that two seed priming treatments significantly enhanced the accumulation of free proline and soluble sugar in soybean seedlings with respect to the unprimed treatment and that the proline content in $\mathrm{CP}$ seedlings was prominently higher than in HP under soda saline-alkali stress (Table 2). These results are consistent with $\mathrm{Hu}$ et al. (2006), who reported that higher seed germination and seedling growth after seed priming were associated with an increase of soluble sugar and proline concentrations in alfalfa under salinity stress. Indeed, a positive correlation between proline accumulation and salt tolerance can be used as an index of salt tolerance among plants (Misra and Gupta, 2005).

Abiotic stress leads to the overproduction of reactive oxygen species (ROS) in plants, which can cause the degradation of proteins and nucleic acids, peroxidation of lipids and MDA production, and then destroy normal cellular metabolism (Gill and Tuteja, 2010). The increase of antioxidant capacity improves the ability of plants to scavenge ROS and to withstand abiotic stress. Superoxide dismutase is ubiquitous and the most effective intracellular enzymatic antioxidant, which provides the first line of defense against the toxic effects of elevated levels of ROS and contributes to the improvement of plant stress tolerance. Catalases with higher turnover rates can convert approximately 6 million molecules of $\mathrm{H}_{2} \mathrm{O}_{2}$ to $\mathrm{H}_{2} \mathrm{O}$ and $\mathrm{O}_{2}$ per minute only using one molecule of CAT (Gill and Tuteja, 2010). In the present study, soybean seedlings had remarkable higher SOD and CAT activities and lower MDA content in CP than HP (Table 3). These results reflect that after priming seeds with comprehensive agents, soybean seedlings strengthen the ability of eliminating ROS through enhancing activities of the antioxidant enzyme system. Younesi and Moradi (2015) also proved that there is a negative relationship between antioxidant enzyme activity and MDA.

The photosynthetic pigments were accumulated in sunflower seedlings, and seeds were inoculated with plant growth promoting rhizobacteria under water stress (Singh et al., 2015). Similar results of pigment accumulation were obtained in our research. Chlorophyll a, chlorophyll b, carotenoids and total pigment contents in soybean seedlings from primed treatments under soda saline-alkali stress were significantly increased compared to the unprimed treatment. Moreover, all of the pigments were remarkably more from seedlings of CP than those of HP and UP (Figure 1). This resulted in seedlings from $\mathrm{CP}$ having more added pigments that might be attributable to $\mathrm{ZnSO}_{4}$, which was consistently used in the priming agents. Zinc is essential for several biochemical processes in plants and can increase membrane integrity, cytochrome and nucleotide synthesis, synthesis of carbohydrates, auxin synthesis, chlorophyll synthesis and metabolism of N (Marschner et al., 1996).

In the present study, the ultrastructural changes that occurred in the mesophyll cells of different treatments were found to vary in damage degree. The mesophyll cells were significantly damaged from UP and HP soybean seedlings because they were sawn to happen the plasmolysis phenomenon (Figures 2A-2D). Under soda saline-alkali stress, soybean seedlings showed induced water absorption difficulty because of a lower water potential around the root systems. Naidoo et al. (2011) likewise reported that the plasma membrane of palisade cells was withdrawn from the cell wall in the mangrove Avicennia marina when in a hypersaline surrounding. Yet, the mesophyll cells of seedlings from seeds treated by $\mathrm{CP}$ did not have the plasmolysis phenomenon. Plastoglobuli may be induced by lipid release upon thylakoid destruction caused ROS under salt stress (Barhoumi et al., 2007). Plastoglobuli from UP seedlings were generally greater in number and size in chloroplasts from the leaves than those from CP (Figures 2A, 2B, 2E and 2F), which demonstrated destruction of the thylakoid was lower and the photosynthetic capacity of mesophyll cells was stronger in soybean seedlings from CP. In abiotic stresses, there are various results regarding the starch accumulation in many types of research. The sensitive cultivar of $B$. napus formed a larger starch grain size than others under chromium stress (Gill et al., 2015). There were many starch grains in the chloroplasts of alpine plants, which were considered an adaptation to the low temperature of the alpine environment (He et al. 2005). In this study, more starch grains were found in the chloroplasts of spongy mesophyll cells from CP than UP and HP (Figures 2A, 2C and 2E). Starch accumulation may be considered an increase of the photosynthetic activity and/or the inhibition of carbohydrate transport from the leaves to other organs. However, for plants, a higher concentration of sugar was accumulated in the chloroplasts and close to the thylakoids, which can avoid thylakoid degradation to maintain normal photophosphorylation 
under low temperatures (He, 1995). Therefore, the leaf ultrastructural results suggest that the mesophyll cells from CP treatment were damaged slightly more under soda saline-alkali stress.

\section{CONCLUSION}

In general, seed priming treatments, including hydropriming and comprehensive seed priming, can mitigate the effects of soda saline-alkali stress in soybean seedlings. Moreover, seed priming with $\mathrm{ZnSO}_{4}, \mathrm{CaCl}_{2}$, betaine hydrochloride and $\mathrm{GA}_{3}$ was found to be more effective than hydropriming to thrive under soda saline-alkali stress. The better growth performance of soybean seedlings from comprehensive seed priming was associated with a stronger osmotic adjustment, higher activities of the antioxidant defense system, more photosynthetic pigment contents, better membrane integrity and more added starch accumulation under soda salinealkali stress. However, further research is necessary to determine the molecular mechanisms of comprehensive seed priming-induced soda saline-alkali tolerance.

\section{ACKNOWLEDGEMENTS}

This research was supported by a grant from China's Soybean System Project (nr CARS-04-PS17), the National Natural Science Foundation of China (nr 31401332, 204134008) and the National Science Technology Support Plan (nr 2014BAD11B01-02). Soybean cv. Nematode resistance No. 10 was kindly donated by the Daqing Branch of Heilongjiang Academy of Agricultural Sciences, Heilongjiang Province, China.

\section{REFERENCES}

Abraha, B., and Yohannes, G. 2013. The role of seed priming in improving seedling growth of maize (Zea mays L.) under salt stress at field conditions. Agricultural Sciences 4:666-672.

Aloui, H., Souguir, M., Latique, S., and Hannachi, C. 2014. Germination and growth in control and primed seeds of pepper as affected by salt stress. Cercetari Agronomice in Moldova 47:83-95.

Ashraf, M., and Foolad, R.M. 2005. Pre-sowing seed treatment a shotgun approach to improve germination, plant growth and crop yield under saline and non-saline conditions. Advances in Agronomy 88:223-271.

Barhoumi, Z., Djebali, W., Chaibi, W., Abdelly, C., and Samaoui, A. 2007. Salt impact on photosynthesis and leaf ultrastructure of Aeluropus littoralis. Journal of Plant Research 120:529-537.

Bradford, M.M. 1976. A rapid and sensitive method for the quantitation of microgram of protein utilizing the principle of protein-dye binding. Analytical Biochemistry 72:248-254.

Dai, L.Y., Yin, K.D., Li, Z.J., and He, S.P. 2015. Chinese Patent nr ZL 201410142570.4.

Dai, L.Y., Zhang, L.J., Ruan, Y.Y., Fan, J.J., and Hu, K. 2012. Effects of saline sodic stress on the blade structure and resistant indexes in sweet sorghum (Sorghum bicolor L. Moench). Journal of Agro-Environment Science 31:468-475. Chinese.
Gill, S.S., and Tuteja, N. 2010. Reactive oxygen species and antioxidant machinery in abiotic stress tolerance in crop plants. Plant Physiology and Biochemistry 48:909-930.

Gill, R.A., Zang, L., Ali, B., Faroog, M., Cui, P., Yang, S., et al. 2015. Chromium-induced physio-chemical and ultrastructural changes in four cultivars of Brassica napus L. Chemosphere 120:154-164.

Guo, Q., Wang, T., Wang, T., and Liang, Y. 2008. Comparative study on leaf ultrastructure in varieties of Chrysanthemum morifolium. Chinese Journal of Chinese Materia Medica 33:10-14. Chinese.

Guo, W-Q, Zhang, P.T., Li, C.H., Yin, J.M. and Han, X-Y. 2015. Recovery of root growth and physiological characters in cotton after salt stress relief. Chilean Journal of Agricultural Research 75:85-91.

Harris, D., Rashid, A., Miraj, G., Arif, M., and Yunas, M. 2008. 'On-farm' seed priming with zinc in chickpea and wheat in Pakistan. Plant and Soil 306:3-10.

He, R.Y. 1995. Plant physical response to low temperature stress. p. 107-141. China Agriculture Press, Beijing, China.

He, T., Wu, X., Zhang, G., Wang, X., and Jia, J. 2005. Characteristics of starch grains in chloroplast of five alpine plants. Journal of Wuhan Botanical Research 23:545-548. Chinese.

Hu, J., Xie, X.J., Wang, Z.F., and Song W.J. 2006. Sand priming improves germination and growth of alfalfa under high-salt concentration stress. Seed Science and Technology 34:199-204.

Hussain, S., Khan, F., Hussain, H.A., and Nie, L. 2016. Physiological and biochemical mechanisms of seed priming-induced chilling tolerance in rice cultivars. Frontiers in Plant Science 7:116.

Ibrahim, E.A. 2016. Seed priming to alleviate salinity stress in germinating seeds. Journal of Plant Physiology 192:38-46.

Kaya, M.D., Okçub, G., Ataka, M., Çıkılıc, Y., and Kolsarıcıa, Ö. 2006. Seed treatments to overcome salt and drought stress during germination in sunflower (Helianthus annuus L.) European Journal Agronomy 24:291-295.

Khan, H.A., Pervez, M.A., Ayub, C.M., Ziaf, K., Bilal, R.M., Shahid, M.A., et al. 2009. Hormonal priming alleviates salt stress in hot pepper (Capsicum annuum L.). Soil and Environment 28:130-135.

Korkmaz, A., and Şirikçi, R. 2011. Improving salinity tolerance of germinating seeds by exogenous application of glycinebetaine in pepper. Seed Science and Technology 39:377-388.

Lichtenthaler, H.K. 1987. Chlorophyll and carotenoids: pigments of photosynthetic bio-membranes. p. 350-382. In Packer, L., and Douce, R. (eds.) Methods in enzymology. Academic, Sandiego.

Liu, Y., Bino, R.J., Van, D., Burg, W.J., Groot, S.P.C., and Hilhorst, H.W.M. 1996. Effects of osmotic priming on dormancy and storability of tomato (Lycopersicon esculentum Mill.) seeds. Seed Science Research 9:49-55.

Marschner, H., Kirkby, E.A., and Cakmak, I. 1996. Effect of the nutritional status on shoot-root partitioning of photoassimilates and cycling of mineral nutrients. Journal of Experimental Botany 47:1255-1263.

Miladinov, Z.J., Balesevic-Tubic, S.N., Dordevic, V.B., Dukic, V.H., Ilic, A.D., and Cobanovic, L.M. 2015. Optimal time of soybean seed priming and primer effect under salt stress conditions. Journal of Agricultural Science, Belgrade 60:109-117.

Miransari, M., and Smith, D.L. 2014. Plant hormones and seed germination. Environmental and Experimental Botany 99:110-121.

Misra, N., and Gupta, A.K. 2005. Effect of salt stress on proline metabolism in two high yielding genotypes of green gram. Plant Science 169:331-339. 
Misra, A.N., Srivastava, A., and Strasser, R.J. 2001. Utilization of fast chlorophyll $a$ fluorescence technique in assessing the salt/ ion sensitivity of mung bean and Brassica seedlings. Journal of Plant Physiology 158:1173-1181.

Naidoo, G., Hiralal, O., and Naidoo, Y. 2011. Hypersalinity effects on leaf ultrastructure and physiology in the mangrove Avicennia marin. Flora 206:814-820.

Nazarli, H., and Faraji, F. 2011. Response of proline, soluble sugars and antioxidant enzymes in wheat (Triticum aestivum L.) to different irrigation regimes in greenhouse condition. Cercetări Agronomice în Moldova 4:27-33.

Paparella, S., Araújo, S.S., Rossi, G., Wijayasinghe, M., Carbonera, D., and Balestrazzi, A. 2015. Seed priming: state of the art and new perspectives. Plant Cell Reports 34:1281-1293.

Patade, V.Y., Maya, K., and Zakwan, A. 2011. Seed priming mediated germination improvement and tolerance to subsequent exposure to cold and salt stress in capsicum. Research Journal of Seed Science 4:125-136.

Pradhan, N., Prakash, P., Tiwari, S.K., Manimurugan, C., Sharma, R.P., and Singh, P.M. 2014. Osmopriming of tomato genotypes with polyethylene glycol 6000 induces tolerance to salinity stress. Trends in Biosciences 7:4412-4417.

Shafi, M., Bakht, J., Hassan, M.J., Raziuddin, M., and Zhang, G. 2009. Effect of cadmium and salinity stresses on growth and antioxidant enzyme activities of wheat (Triticum aestivum L.) Bulletin of Environmental Contamination and Toxicology 82:772-776.
Singh, N.B., Singh, D., and Singh, A. 2015. Biological seed priming mitigates the effects of water stress in sunflower seedlings. Physiology and Molecular Biology of Plants 21:207-214.

Younesi, O., and Moradi, A. 2015. Effect of priming of seeds of Medicago sativa 'bami' with gibberellic acid on germination, seedlings growth and antioxidant enzymes activity under salinity stress. Journal of Horticultural Research 22:167-174.

Zheng, S.L., Cheng, H., Li, P.H., and Yuan, J.C. 2016. Root vigor and kinetic characteristics and nitrogen use efficiencies of different potato (Solanum tuberosum L.) cultivars. Journal of Agricultural Science and Technology 18:399-410.

Zheng, M., Hussain, S., Tao, Y., Jiang, Q., Peng, S., Huang, J., et al. 2015. Seed priming in dry direct-seeded rice: consequences for emergence, seedling growth and associated metabolic events under drought stress. Plant Growth Regulation 78:167-178.

Zhu, S., Zhang, X., Luo, T., Liu, Q., Tang, Z., and Jing, Z. 2011. Effects of $\mathrm{NaCl}$ stress on seed germination, early seedling growth and physiological characteristics of cauliflower (Brassica oleracea L. var. botrytis L.) African Journal of Biotechnology 10:17940-17947. 